\title{
Signaling the omission of a response-contingent outcome reduces discriminative control
}

\author{
RUTH M. COLWILL \\ Brown University, Providence, Rhode Island
}

\begin{abstract}
The effect of training a positive discriminative stimulus $(\mathrm{S}+)$ as a signal for the nonreinforcement of an instrumental response (S-) on the ability of that stimulus to evoke its original instrumental response was examined in three experiments using rats. In all three experiments, two different stimuli were established as $\mathrm{S}+\mathrm{s}$ for different response-outcome relations. In Experiment 1 , an $\mathrm{S}+$ was less effective in controlling its original response after it had undergone training as an $\mathrm{S}-$ for a new response that earned the same outcome than it was after training as an $\mathrm{S}-$ for a response that earned a different outcome. Experiment 2 established that this effect was not mediated by Pavlovian inhibitory conditioning produced by the negative correlation between the $\mathrm{S}+$ and the outcome during $\mathrm{S}-$ training. Simply arranging a negative correlation between $\mathrm{S}+$ and the outcome whose occurrence it had previously signaled did not impair the ability of that S+ to elicit its original response. In Experiment 3, the response-evoking properties of an S+ were found to be undermined by using the $\mathrm{S}+$ as a signal for the simple extinction of a new response trained with the same outcome, but not with a different outcome. These results suggest that positive discriminative stimuli use their associations with the outcomes earned in their presence to control the responses that earned those outcomes.
\end{abstract}

Classical S-R theory provides a simple explanation for discriminative instrumental performance (e.g., Hull, 1943). A discriminative stimulus ( $\mathrm{S}+$ ) is thought to evoke its instrumental response ( $R$ ) by virtue of an association between the $\mathrm{S}+$ and the $\mathrm{R}$. That $\mathrm{S}-\mathrm{R}$ association is assumed to be strengthened whenever the responsecontingent outcome $(\mathrm{O})$ follows contiguities of the stimulus and the response. According to this view, instrumental performance depends primarily upon the energization of an intact S-R association.

Recent studies of instrumental learning have identified some serious shortcomings in this S-R account of instrumental performance. Contemporary findings suggest that an $\mathrm{S}+$ provides information about the identity of the outcome that is earned in its presence. For instance, Colwill and Rescorla (1988) found that an S+ trained with one response-outcome relation would selectively promote another instrumental response trained with the same outcome but leave unaffected a response trained with a different outcome. The finding that successful transfer is contingent upon shared association with the same outcome suggests that $\mathrm{S}-\mathrm{O}$ associations may contribute to the performance of new instrumental responses. Such a result is obviously quite troublesome for classical S-R theory.

The research reported in this paper was supported by National Science Foundation Grant BNS 89-15342 to the author. I would like to thank Eric Wolfinger and Barbara Shipley for their careful assistance with data collection. Correspondence concerning this article should be addressed to R. M. Colwill, Department of Psychology, Brown University, Box 1853, Providence, RI 02912.
Although these transfer data demonstrate the inadequacy of S-R theory in accounting for the performance of new instrumental responses in the presence of previously established $\mathbf{S}+\mathrm{s}$, the question still remains as to how those $\mathrm{S}+\mathrm{s}$ control their originally trained responses. One possibility suggested by the transfer data is that performance of the original response may be modulated by the $\mathrm{S}-\mathrm{O}$ association in the same way as that of a transfer response. But an equally viable alternative is that the original response is elicited through an S-R association. The present paper used an interference manipulation to assess the potential contributions of $\mathrm{S}-\mathrm{O}$ and $\mathrm{S}-\mathrm{R}$ associations to the ability of an $\mathrm{S}+$ to produce its original instrumental response. The idea behind the manipulation was to reverse the information that $\mathbf{S}+$ had signaled about the availability of the outcome. To the degree that an $\mathrm{S}+$ controls its response through an $\mathrm{S}-\mathrm{O}$ association, it was anticipated that performance of that response would be undermined if the $\mathrm{S}+$ were converted into a signal for the omission of that outcome. On the other hand, the S-R account of discriminative stimulus control would anticipate no impact of that manipulation on the ability of the $S+$ to elicit its original response.

Experiment 1 examined whether an $\mathrm{S}+$ for one $\mathrm{R}-\mathrm{O}$ relation would be affected by training as a signal (S-) for the nonreinforcement of a different response that was otherwise reinforced with the same outcome. Several studies have found that negative discriminative stimuli (S-) encode information about the identity of the omitted response-contingent outcome (Bonardi, 1989; Colwill, 1991). For instance, using the transfer procedure, Colwill (1991) found that an S- produced more substantial 
st training

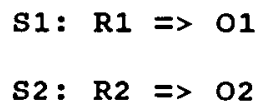

s- training
Test

S1: R1

S2: R2

Figure 1. Basic design of Experiment 1. R1, R2, and R3 are instrumental responses (leverpressing, chainpulling, and nosepoking); $S 1$ and $S 2$ are instrumental discriminative stimuli (noise and light); 01 and $\mathrm{O2}$ are rewarding outcomes (sucrose and pellets).

disruption of a new response trained with the outcome whose omission was predicted by the $S-$ than it did of a response trained with a different outcome. Consequently, it was expected that $S-$ training would establish the former $\mathrm{S}+$ as a signal for the omission of the outcome whose availability it had previously predicted. To the degree that performance of the original response is controlled by the S-O association, this treatment should lead to a reduction in the performance of that response. The results of Experiment 1 confirmed this prediction. Conversion of an $\mathrm{S}+$ to an $\mathrm{S}$ - with the same outcome disrupted the ability of the $S+$ to evoke its original response compared with that of an $\mathrm{S}+$ trained as an $\mathrm{S}-$ with a different outcome. Experiments 2 and 3 explored different explanations for this result and provided support for the view that performance of instrumental responses depends in large part on S-O associations.

\section{EXPERIMENT 1}

The present study was designed to examine the impact on original $\mathrm{S}+$ control of converting an $\mathrm{S}+$ for one response into an $S-$ for a different response trained with either the same or a different outcome. The basic design of Experiment 1 is illustrated in Figure 1. Rats were trained to make two different responses (lever and chain), each for a different outcome (sucrose and pellets), in the presence of two different $S+s$ (light and noise). One response (R1) was reinforced with one outcome $(\mathrm{O} 1)$ in the presence of one stimulus ( $\mathrm{S} 1$ ); the other response (R2) was reinforced with a different outcome $(\mathrm{O} 2)$ in the presence of a different stimulus (S2). Following this training, both stimuli were trained as signals for the nonreinforcement of a new response (R3). For half the animals, $\mathrm{R} 3$ earned $\mathrm{O} 1$ in the absence of the stimuli; for the remaining animals, $\mathrm{R} 3$ earned $\mathrm{O} 2$. Thus, for each animal one $\mathrm{S}+$ signaled the omission of the same outcome used to train that $\mathrm{S}+$ and the other $\mathrm{S}+$ signaled the omission of a different outcome. In this way, any general disruptive effects of $S$ - training were controlled for. In subsequent extinction tests, each $\mathbf{S}+$ was tested with its original response. It was anticipated that the $\mathrm{S}+$ trained as an $\mathrm{S}$ - for the same outcome would be less effective in eliciting its original response (R1 or R2) than would the $S+$ trained as an $\mathrm{S}-$ for a different outcome (R2 or $\mathrm{R} 1$ ).

\begin{abstract}
Method
Subjects

The subjects were 16 experimentally naive Holtzman-derived Sprague-Dawley male rats, about 100 days old at the start of the experiment. They were maintained in individual cages at approximately $80 \%$ of their free-feeding weight. Water was available on an ad-lib schedule.
\end{abstract}

\section{Apparatus}

The apparatus consisted of eight identical Skinner boxes measuring $22.9 \times 20.3 \times 20.3 \mathrm{~cm}$. The two end walls of the chamber were aluminum, and the side walls and ceiling were made of Plexiglas. The floor of the chamber was composed of $0.48-\mathrm{cm}$ stainless steel rods spaced $1.9 \mathrm{~cm}$ apart. Each chamber had a recessed food magazine in the center of one end wall. Sucrose and pellets were delivered through separate tubes inserted through the roof of the food magazine. Each operation of the sucrose dispenser allowed $0.2 \mathrm{ml}$ of $8 \%$ sucrose to flow onto the floor of the food magazine, where it collected in a shallow indentation. Each operation of the pellet dispenser allowed a single $45-\mathrm{mg}$ food pellet (Noyes, Formula A) to drop onto the floor of the magazine. Each box was equipped with a leverpress, a chainpull, a nosepoke, and a handlepull manipulandum. The lever was mounted $2.5 \mathrm{~cm}$ from the right-hand wall of the food magazine. The chain was suspended from a microswitch mounted on the roof of the chamber. The end of the chain was $11 \mathrm{~cm}$ from the grid floor and $3 \mathrm{~cm}$ from the lefthand wall of the food magazine. Located $5.5 \mathrm{~cm}$ directly above the roof of the magazine was the nosepoke manipulandum, which consisted of a circular aperture $2 \mathrm{~cm}$ in diameter and $1.3 \mathrm{~cm}$ deep. The back of this aperture was covered by a metal plate that operated a microswitch whenever it was depressed. Mounted on the same side of the chamber as the chain but $1.5 \mathrm{~cm}$ below the grid floor was the handlepull manipulandum. This consisted of a short, flat rod protruding $3 \mathrm{~cm}$ into the chamber. Whenever the rod was pulled upward, a microswitch was closed and a response recorded. The same model of microswitch (Unimax Switch, 2HBT-1) was used to detect responding on all four manipulanda. Access to these manipulanda was prevented by covering the lever with a metal plate or by retracting it; by retracting the chain through an opening in the ceiling; by inserting a metal cover into the aperture of the nosepoke manipulandum; and by withdrawing the arm of the handlepull. Each Skinner box was enclosed in a sound- and lightresistant shell. Experimental events were controlled and recorded automatically by interfacing (Med Associates) and an XT microprocessor located in an adjoining room.

\section{Procedure}

Magazine training. Subjects received one session of magazine training in which ten $45-\mathrm{mg}$ food pellets (Noyes, Formula A), followed by ten $8 \%$ liquid sucrose reinforcers, were delivered on a variable-time (VT) 60-sec schedule. Operation of both the pellet and the sucrose dispensers was accompanied by a brief click. 
Response training. Following magazine training, all animals were trained to leverpress and chainpull. Initially, each response was trained separately on a continuous-reinforcement (CRF) schedule until 30 reinforcers had been earned. Each response was then reinforced on a variable-interval (VI) 30-sec schedule for three 20-min sessions. Half the animals were trained to leverpress for pellets and chainpull for sucrose; the remaining animals did the reverse.

$S+$ training. Each session contained one of the response manipulanda and 32 presentations of either a $30-\mathrm{sec}$ noise or light discriminative stimulus. Responding was reinforced on a VI $30-\mathrm{sec}$ schedule during an S+ but never during the intertrial interval (ITI). For 8 subjects, the light served as an $S+$ for leverpressing and the noise as an $\mathrm{S}+$ for chainpulling; these stimulus-response combinations were switched for the remaining 8 animals. Within each of these conditions, 4 animals earned pellets for leverpressing and sucrose for chainpulling; the other 4 earned sucrose for leverpressing and pellets for chainpulling. Thus, each subject was trained with one stimulus as an $\mathrm{S}+$ for one response-outcome relation and with another $\mathbf{S}+$ for a different response-outcome relation. There were 10 sessions of $S+$ training with each response. The ITI was gradually increased over sessions: the mean ITI was $15 \mathrm{sec}$ in the first session, $30 \mathrm{sec}$ in the next 3 sessions, $60 \mathrm{sec}$ for the following 3 sessions, and finally, $90 \mathrm{sec}$ for the last 3 sessions, for each response.

S- training. Nosepoking was first trained on a CRF schedule until 30 reinforcers had been earned. This training was followed by three 20 -min sessions of reinforcement on a VI 30-sec schedule. Nosepoking was trained with pellets for half the animals and with sucrose for the other half.

Each session of $S-$ training contained 16 presentations each of the 30 -sec noise and light stimuli, with a mean ITI of $30 \mathrm{sec}$. Nosepoking was reinforced on a VI 30-sec schedule during the ITI but never reinforced during stimulus presentations. The nosepoke response continued to earn the outcome used to train it. Thus, each stimulus was trained as an $S$ - with an outcome that was either the same as (same $S+/ S-$ ) or different from (diff $S+/ S-$ ) that used in the original $S+$ training. There were 15 sessions of this training.

Testing. Four test sessions were conducted, two with the lever and two with the chain. In each test, 4 presentations each of the noise (N) and light (L) stimuli were scheduled, with a mean ITI of $90 \mathrm{sec}$. The order of testing responses was lever then chain on the first day, and chain then lever on the second day. The sequence of test trials was NLLNLNNL on Day 1 and the reverse order on Day 2. Responding was never reinforced during these tests.

\section{Results and Discussion}

Acquisition of the $\mathrm{S}+$ discrimination proceeded smoothly. By the end of training, all animals were responding primarily during the stimuli (18.2 responses per minute), and infrequently during the ITI ( 3.7 responses per minute). Discrimination ratios were calculated for each animal by dividing the response rate during the $\mathrm{S}+$ by the combined rate of responding during the stimulus and during the ITI. The mean discrimination ratio for the final session of training was .84 .

During the initial stages of $S$ - training, there was a tendency for responding to be higher in the presence of the stimulus signaling the omission of the same outcome used during $S+$ training (same $S+/ S-$ ) than it was during the stimulus that signaled omission of a different outcome (diff $\mathrm{S}+/ \mathrm{S}-$ ). The mean discrimination ratios collapsed across the first three sessions were .52 and .45 for the same and different conditions, respectively. This difference narrowly escaped conventional levels of signifi- cance [Wilcoxon $T(16)=32, p<.10$ ]. This finding is in line with other work showing that an $S+$ will promote performance of other instrumental responses trained with the same outcome (Colwill \& Rescorla, 1988). The circumstances under which the present difference was obtained differ considerably from those of the original transfer experiments. Of most relevance is that those studies used a more sensitive choice-extinction test. This difference may well account for the greater difficulty in detecting transfer that was observed in the present study. With continued $S-$ training, the difference between the same and different $S+/ S-$ conditions disappeared. The mean discrimination ratios for the final session of $S-$ training were .21 and .25 for the same and different conditions, respectively $[T(16)=52, p>.10]$.

The results of most interest concern the extinction tests with the lever and chain, and are depicted in Figure 2. Performance of each response is shown during the stimulus with which it was trained and during the ITI. The left side of Figure 2 displays responding in the presence of the same $\mathrm{S}+/ \mathrm{S}-$; the right side shows responding during the different $S+/ S-$. It is clear that the stimuli differ markedly in their ability to control their originally trained response. Discrimination ratios were calculated for each subject for the same and different $S+s$. The same $S+/ S-$ produced significantly less elevation than did the different $S+/ S-[.52$ and .69 , respectively; $T(16)=11.5, p<$ .01 ]: Moreover, compared with the ITI rates, the same $\mathrm{S}+/ \mathrm{S}-$ did not produce significant elevation of its response $[T(16)=59, p>.10]$, whereas the different

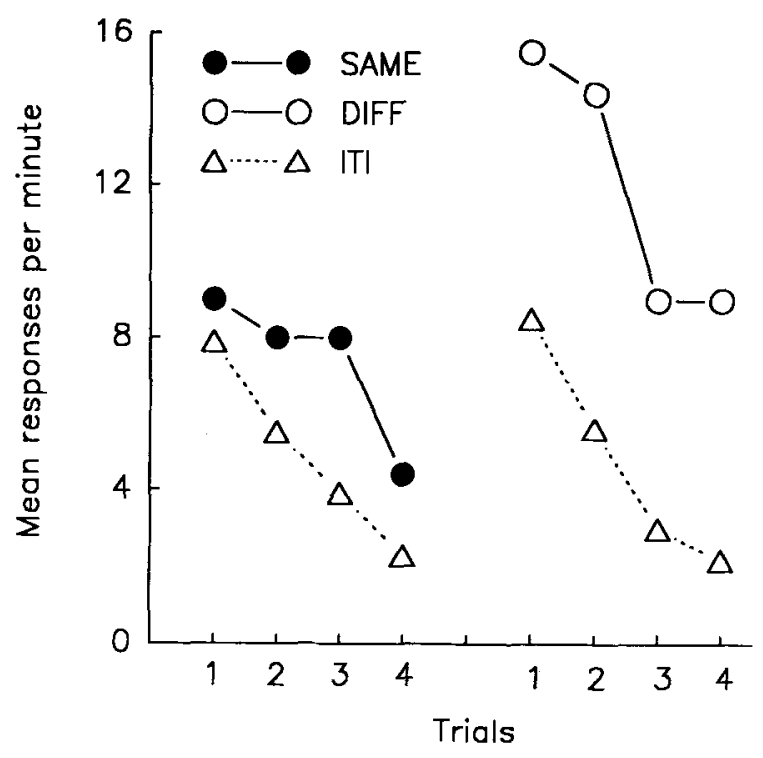

Figure 2. Experiment 1: Mean responses per minute during the first pair of extinction tests, in which each $\mathbf{S}+$ was presented with its original response. To the left, responding is shown during the $S+$ that was trained as an S- with the same outcome (filled circles); to the right, responding is shown during the $S+$ that was trained as an $S-$ with a different outcome (open circles). Responding during the ITIs is also shown (triangles). 
$\mathrm{S}+/ \mathrm{S}-$ continued to produce a significant increase in its response $[T(16)=1, p<.01]$.

The second pair of test sessions confirmed these findings. Mean discrimination ratios for the same and different $S+/ S-s$ indicated that the same $S+/ S-$ was less effective in producing its response (.59) than was the different $\mathrm{S}+/ \mathrm{S}-[.77 ; T(16)=22, p<.05]$. Furthermore, the mean rate of responding during the same $\mathrm{S}+/ \mathrm{S}-(6.9$ responses per minute) was not significantly different from its ITI rate $[3.0$ responses per minute; $T(16)=40.5, p>.10$ ], whereas responding during the different $S+/ S-(9.6$ responses per minute) was significantly greater than its ITI rate $[2.6$ responses per minute; $T(16)=0, p<.01]$.

These results demonstrate that the ability of an $\mathrm{S}+$ to produce its original response may be undermined by training that $\mathrm{S}+$ as a signal for the nonreinforcement of a different response that earns the same outcome. The evidence that this impairment depends on outcome identity implicates the outcome in the control of the original response. Thus, an account of instrumental performance exclusively in terms of $\mathrm{S}-\mathrm{R}$ associations would seem to be inadequate for explaining responding in the presence of an $\mathrm{S}+$.

\section{EXPERIMENT 2}

There are several features of $\mathrm{S}$ - training that may have been responsible for undermining original responding in the presence of $\mathbf{S}+$. One obvious possibility is that the discriminative stimulus acquired Pavlovian inhibitory properties as a result of the negative correlation between the $\mathrm{S}+$ and the outcome that is embedded in $\mathrm{S}-$ training. It has been well established that such negative correlations can endow stimuli with the ability to suppress responding to Pavlovian excitors (Rescorla, 1969) or to produce withdrawal from the negative stimulus (Hearst \& Franklin, 1977). Of even more interest, it has been demonstrated that in aversive conditioning procedures stimuli negatively correlated with an outcome may suppress instrumental responses maintained by that outcome (e.g., Rescorla \& LoLordo, 1965; Weisman \& Litner, 1969).

The purpose of Experiment 2 was to evaluate this conditioned inhibition account of the attenuation of $\mathrm{S}+$ control found in Experiment 1. To this end, it examined the consequences of arranging a simple negative correlation between the $\mathrm{S}+$ and the outcome. The basic design, shown in Figure 3, was modeled after that of Experiment 1. Two stimuli were established as $\mathrm{S}+\mathrm{s}$, each for a different response-outcome relation. Then both stimuli were negatively correlated with one of the two outcomes. Finally, each stimulus was tested in extinction with its original response. To the degree that the loss of S+ control observed in the previous studies was mediated by Pavlovian inhibition, it was anticipated that arranging a negative correlation between $\mathrm{S}+$ and $\mathrm{O}$ would interfere with responding to the S+ trained with the same outcome.

\section{Method}

\section{Subjects and Apparatus}

Sixteen experimentally naive Holtzman-derived Sprague-Dawley male rats, about 100 days old, served as subjects. They were maintained under the same conditions as the subjects in Experiment 1. The apparatus used was that of Experiment 1.

\section{Procedure}

Magazine, response, and S+ training. The details of these procedures are identical to those described in Experiment 1.

Negative correlation training. During this phase, no response manipulanda were available. In each session, there were 16 nonreinforced presentations of each $\mathrm{S}+$, with an ITI of $30 \mathrm{sec}$. During the ITI, one of the outcomes was delivered on a VT 30-sec schedule. For half the animals, pellets served as the ITI outcome; for the remaining animals, sucrose served as the ITI outcome. There were 15 sessions of this training, thus matching the amount of $S-$ training administered in Experiment 1.

Testing. Subjects were tested according to the same procedure described in Experiment 1. Four test sessions were conducted, two with the lever and two with the chain. In each test, 4 presentations each of the noise and light stimuli were scheduled, with a mean ITI of $90 \mathrm{sec}$. The order of testing responses was lever then chain on the first day, and chain then lever on the second day. The sequence of test trials was NLLNLNNL on Day 1 and the reverse order on Day 2. Responding was never reinforced during these tests.

\section{Results and Discussion}

All subjects acquired the initial $\mathrm{S}+$ discrimination. By the end of training, the rate of responding was substantially higher during the noise and light $S+s$ (19.7 responses per minute) than during the ITI (4.0 responses per $\mathrm{min}$ ). The mean discrimination ratio for the final session was .86 .

The findings of most importance from the first test day are displayed in Figure 4. Leverpressing and chainpulling in the presence of the $S+s$ with which they were trained are shown as a function of whether those $S+s$ were established as Pavlovian inhibitors for the same outcome (left side of figure) or the different outcome (right side). 


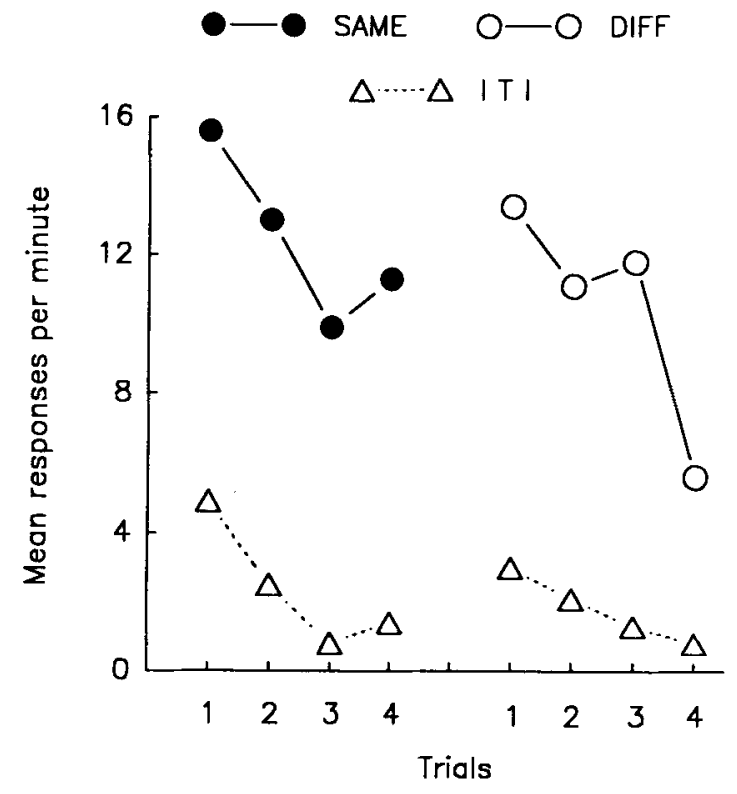

Figure 4. Experiment 2: Mean responses per minute during the test of the $\mathrm{S}+\mathrm{s}$ with their original responses. Responding during the stimulus that was negatively correlated with the same outcome used for initial S+ training is shown on the left; responding during the stimulus that was negatively correlated with a different outcome from the one used for $\mathbf{S}+$ training is shown on the right. Responding during the ITIs is also shown (triangles).

In both conditions, the stimuli continued to produce their instrumental responses. Comparison of the response rates during the stimuli showed no significant difference between the same and different conditions during the first test $[T(15)=54, p>.10]$. Overall discrimination ratios were calculated and compared. There was no significant difference between the same $(.81)$ and different $(.82)$ conditions $[T(15)=51.5, p>.10]$. Similar results were obtained on the second test day. The mean rate of responding produced by the same $\mathrm{S}+$ was again slightly higher than that produced by the different $S+(9.6$ and 8.4 responses per minute, respectively). That difference was also apparent in the ITI rates (1.5 and 1.1 responses per minute, respectively). However, in neither case were these differences between the same and different conditions statistically significant.

In this experiment, no loss of discriminative control was obtained when $\mathrm{S}+$ was simply negatively correlated with the outcome whose occurrence it had previously signaled. This result suggests that the reduction observed in Experiment 1 in the ability of an $S+$ to produce its response was not a consequence of exposure to the negative correlation between that $\mathbf{S}+$ and its outcome during $\mathbf{S}$ - training. This finding is consistent with several reports that stimuli trained as signals for the absence of a food outcome in either differential or Pavlovian conditioned inhibition procedures do not suppress instrumental responding maintained by that food outcome (e.g., Lovibond, 1983; Wielkiewicz, 1979).

\section{EXPERIMENT 3}

Experiment 2 isolated a potential source of Pavlovian conditioned inhibition embedded in $\mathbf{S}-$ training and found that it did not contribute to the differential loss of discriminative control observed in Experiment 1. In Experiment 3 , the role of signaling the nonreinforcement of a response was examined under circumstances that removed any contamination by potential Pavlovian associations between $\mathrm{S}+$ and the negatively correlated $\mathrm{O}$. This was accomplished by making $S+$ signal the extinction of another instrumental response that had been trained with either the same or a different outcome. On the basis of the findings of Experiments 1 and 2, it was anticipated that differential loss of discriminative control would be obtained.

Rats were first trained to make two different instrumental responses, leverpress and chainpull $(R 1$ and $R 2$, respectively), for two different outcomes, sucrose and pellets (O1 and $\mathrm{O} 2)$, in the presence of each of two different discriminative stimuli, noise and light (S1 and S2). Then two new responses, nosepoke and handlepull, were trained, one, R3, with $\mathrm{O} 1$ and the other, $\mathrm{R} 4$, with $\mathrm{O} 2$. Following training, these responses were nonreinforced in the presence of the two discriminative stimuli: R3 was extinguished in the presence of S1 and R4 in the presence of S2. Finally, R1 and R2 were made available in an extinction test with periodic presentations of $\mathrm{S} 1$ and $\mathrm{S} 2$. This design is outlined in Figure 5.

In addition to evaluating the specific contribution of response extinction to the results of Experiment 1, Experiment 3 has some general implications for theories of extinction. It has been typical for accounts of extinction in both Pavlovian and instrumental conditioning procedures to appeal to decremental processes that do not take the identity of the outcome into consideration (e.g., Amsel, 1962; Capaldi, 1970; Robbins, 1990). Thus, based on existing theories of extinction, the identity of the outcome used to train the to-be-extinguished response should be irrelevant in the present experiment. However, if extinction involves learning about the specific outcome that will not occur, then performance of $\mathrm{R} 1$, the response that was trained with $\mathrm{O1}$, should be selectively depressed in S1, and performance of $\mathrm{R} 2$, the response that was trained with $\mathrm{O} 2$, should be selectively depressed in S2. Consequently, if the reduction in discriminative control produced by signaling the extinction of a response were found to depend upon the identity of the outcome used to train the response, current theories of extinction would need to be revised.

\section{Method}

\section{Subjects and Apparatus}

Sixteen experimentally naive Holtzman-derived Sprague-Dawley male rats, approximately 100 days old, served as subjects. They were maintained under the same conditions as the subjects in Experiment 1. The apparatus used was that of Experiment 1.

\section{Procedure}

Magazine and response training. All subjects received one session of magazine training, in which 10 pellet and 10 sucrose rein- 
s+ training
Extinction

Test

$$
\begin{aligned}
& R 3=>01 \\
& R 4=>02
\end{aligned}
$$
S1: R1 $=>01, R 2 \Rightarrow 02$
S1: R3-
S2: R4-
S1: R1 V R2
S2: R1 V R2

\begin{abstract}
Figure 5. Basic design of Experiment 3. R1, R2, R3, and R4 are instrumental responses (leverpressing, chainpulling, nosepoking, and handlepulling); $S 1$ and $S 2$ are discriminative stimuli (noise and light); 01 and $\mathbf{O 2}$ are reinforcers (sucrose and pellets).
\end{abstract}

forcers were delivered on a VT 60 -sec schedule. Each subject was then trained to leverpress and chainpull. Initially, each response was reinforced on a CRF schedule until 30 reinforcers had been obtained. Each of these responses was then trained on a VI 30-sec schedule for two 20 -min sessions. For 8 subjects, leverpress responses earned pellets and chainpull responses earned sucrose; for the other 8 subjects, the opposite response-outcome combinations were arranged.

S+ training. There were 9 sessions of training, with each response arranged in a double alternating schedule. Each session contained sixteen 30 -sec presentations each of a light and a noise stimulus. Responding was reinforced on a VI 30-sec schedule during the stimuli with the same outcome as was used for its initial training. The mean ITI was gradually increased from $30 \mathrm{sec}$ to $90 \mathrm{sec}$ across sessions. The mean ITI was $30 \mathrm{sec}$ for the first 4 sessions of training with each response; it was increased to $60 \mathrm{sec}$ for the next 3 sessions with each response; and it was set at $90 \mathrm{sec}$ for the final 2 sessions with each response.

Extinction. Two new responses, nosepoke and handlepull, were reinforced, one with pellets and one with sucrose. Initially, subjects were permitted to earn 30 reinforcers, first for nosepoking and then, in a separate session, for handlepulling. Following this, each response was trained separately on a VI 30 -sec schedule for four 20-min sessions.

These responses were then extinguished in the presence of the $S+s$. Each of the extinction sessions contained one response and 8 presentations of either the noise or the light $S+s$. The ITI was $90 \mathrm{sec}$. Responding was never reinforced in these sessions. There were 2 extinction sessions per day, one with each response, for 6 days. One 20 -min session of VI 30 -sec training was given with both the nosepoke and the handlepull responses after the first test session administered between Days 4 and 5 of the extinction treatment. Thus, for each subject, one $S+$ signaled the extinction of a pellet-trained response and the other $S+$ signaled the extinction of a sucrose-trained response.

Testing. The lever and chain were tested in extinction with 4 presentations each of the noise and light $S+s$, with an ITI of 90 sec. The first of these tests was conducted after Day 4 of $S+$ extinction, and the second test was after Day 6 . The order of stimulus presentations was NLLNLNNL. The stimulus sequence was reversed for the second test session.

\section{Results and Discussion}

Over the course of $\mathbf{S}+$ training, all but two of the subjects came to respond primarily during the noise and light stimuli. Of these two, one failed to make the sucrosetrained response in the presence of either $S+$, and the other responded as much during the ITI as during the $\mathrm{S}+\mathrm{s}$. Consequently, the data from these subjects were discarded from all subsequent analyses. For the remaining subjects, the mean rate of responding in the final session of $\mathrm{S}+$ training, collapsed across response, outcome, and stimulus identity, was 18.3 responses per minute in the presence of the $\mathrm{S}+\mathrm{s}$ and 3.7 per minute during the ITI $[T(14)=0, p<.01]$. Mean discrimination ratios were calculated for each animal by dividing the rate of responding during the $\mathbf{S}+\mathbf{s}$ by the sum of that rate and the response rate during the ITI. The mean ratio across animals was .85 .

Acquisition of the nose-poke and handle-pull responses proceeded uneventfully. The mean rate of responding for the final VI training session, collapsed across response and reinforcer identity, was 16.8 responses per minute. Assignment of the response to be extinguished in a particular S+ was counterbalanced across animals with respect to the various stimulus, response, and outcome combinations. Care was taken to ensure that the differences the extinction treatment was expected to produce were not present at the end of discrimination training. Analysis of the last $\mathrm{S}+$ training session showed that the mean discrimination ratios for the responses trained either with the outcome that was the same as that earned by the extinguished response or with the outcome that was different were not significantly different ( .86 and .85 , respectively). Over the course of signaled extinction, performance of the nosepoke and handlepull responses declined. The mean response rates in the presence and absence of the $\mathrm{S}+\mathrm{s}$ were 11.4 and 9.2 responses per minute in Session 1; 3.3 and 3.7 responses per minute in Session 4; and 1.7 and 2.0 responses per minute in the final session of extinction.

The results of most interest concern the effects of the extinction treatments on the ability of the $S+s$ to produce their original responses. The data from the first test session were moderately encouraging. The mean rate of responding trained with the same outcome as that earned by the extinguished response was lower than that trained with a different outcome (8.0 and 9.6 responses per minute, respectively), but this difference was not statistically significant $[T(14)=33, p>.05]$. However, a reliable difference did emerge following additional extinction sessions. The results of the second test session are displayed in Figure 6. Responding is plotted as a function of whether the response extinguished in the $S+$ earned the same reinforcer or a different reinforcer. Inspection of Figure 6 reveals that responding was selectively reduced by extinction of another response that had earned 


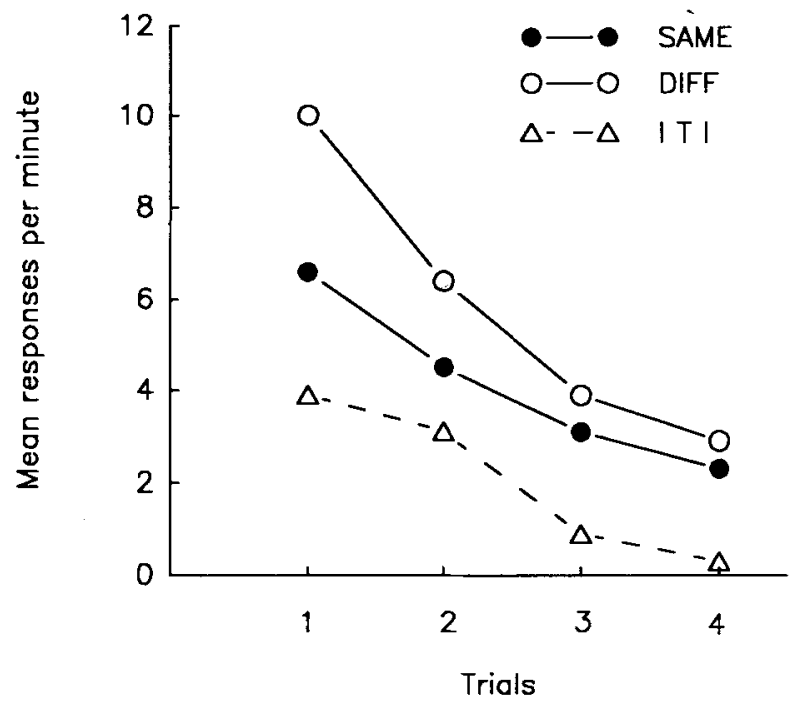

Figure 6. Experiment 3: Mean responses per minute during the second test session with the lever and chain. Responding during the ITI is shown (triangles), and also that during each $S+$ as a function of whether that $\mathbf{S}+$ had signaled extinction of another response trained either with the same outcome (filled circles) or with a different outcome (open circles).

the same outcome. An overall analysis of the second test found a significant difference between the same and different conditions $[T(13)=13.5, p<.05]$.

Further analysis of the data from the second test session indicated that the extinction treatment had undermined the ability of the $\mathbf{S}+$ to elevate the same response relative to that during the ITI $[T(14)=38.5, p>.10]$. In contrast, performance of the different response continued to be higher during the $\mathrm{S}+$ than it was during the ITI $[T(14)=10, p<.01]$.

These results confirm and extend those of Experiment 1 . In both experiments, the effectiveness of an $\mathrm{S}+$ in controlling its original response was reduced by establishing that $S_{+}$as a signal that another instrumental response would not be reinforced with that outcome. Furthermore, Experiment 3 found a loss of $\mathrm{S}+$ control under circumstances that did not involve differential experience with one of the outcomes. In both Experiments 1 and 2, only one of the outcomes was presented during the interference treatment. Those outcomes were responsecontingent in the first experiment but freely delivered in the second. The fact that $S+$ control was not eliminated in Experiment 2 but was in Experiment 3 suggests that differential experience with the outcome per se or with an earned outcome is not the aspect of $S-$ training that is responsible for the decrement in $\mathrm{S}+$ control. Thus, the ability of an $\mathrm{S}+$ to control its original response is not adversely affected by separate presentations of the outcome whose availability it has previously signaled.

The finding that nonreinforcement of an instrumental response in the presence of an $\mathrm{S}+$ produces a loss of $\mathrm{S}+$ control emphasizes the need for theories of extinction to incorporate an outcome-specific component. Only if information about the identity of the omitted outcome had been generated could the loss of discriminative control have been dependent on outcome identities. Thus, the present findings are awkward for any theory of nonreinforcement that appeals exclusively to nonspecific decremental processes to account for reduced responding (e.g., Amsel, 1962; Capaldi, 1970).

\section{GENERAL DISCUSSION}

In Experiment 1, the ability of an $\mathrm{S}+$ to evoke its instrumental response was diminished by training that $\mathbf{S}+$ as an $\mathrm{S}-$ for a different response that earned the same outcome. Experiment 2 eliminated one source of Pavlovian conditioned inhibition that might have contributed to this effect. No loss of stimulus control was observed when the $S+$ was simply negatively correlated with the same outcome. These findings suggest that a critical factor in reducing $S+$ control involves training the $S+$ as a signal that responding trained with that outcome will not be reinforced. That conclusion was confirmed in Experiment 3, in which $S+$ control was attenuated by making $S+$ signal the extinction of another instrumental response trained with the same outcome. The results of these studies indicate that an important determinant of the performance of an instrumental response is the information that $\mathrm{S}+$ provides about the availability of the outcome. By signaling that an outcome is available, an $\mathrm{S}+$ is able to promote performance both of its own response and of a new response trained with that outcome.

There have been several speculations about the nature of the learning that enables an $\mathrm{S}+$ to indicate what particular outcome is available. Three different accounts have achieved prominence. Two of these accounts derive from a binary analysis of instrumental learning; the third emerges from an attempt to embrace within a single associative structure the three elements of an instrumental conditioning procedure. Each account is of interest because it suggests a different explanation for the disruptive effect on S+ control that results from making $\mathrm{S}+$ signal the nonreinforcement of a response trained with the same outcome.

Several authors have argued that a Pavlovian association develops between the discriminative stimulus and the instrumental outcome. That Pavlovian association has been assumed either to motivate the instrumental response (Rescorla \& Solomon, 1967) or to provide stimulus support for the response (Trapold \& Overmier, 1972). Given either one of these theoretical orientations, it makes sense to view the operations used in Experiments 1 and 3 as encouraging the development of an inhibitory $\mathrm{S}-\mathrm{O}$ association. In both of those experiments, $\mathrm{S}+$ signaled the omission of an expected outcome. Studies of Pavlovian conditioning have shown this procedure to be very effective in training a stimulus as an inhibitor (see Mackintosh, 1983; Rescorla, 1979). There are several ways in which this kind of inhibitory learning might interfere with the 
original $\mathrm{S}-\mathrm{O}$ association. For instance, the $\mathrm{S}-\mathrm{O}$ association may be masked by a competing $\mathrm{S}-$ no- $\mathrm{O}$ association. On the other hand, the original $\mathrm{S}-\mathrm{O}$ association may be neutralized by a parallel inhibitory $S-O$ association. Finally, the excitatory $\mathrm{S}-\mathrm{O}$ association may simply be erased by procedures that generate inhibition. The virtues of these different views of conditioned inhibition have been considered elsewhere (e.g., LoLordo \& Fairless, 1985; Rescorla, 1979).

At first, it may seem as if the results of Experiment 2 are incompatible with this sort of conditioned inhibition analysis of the loss of S+ control. For instance, several studies have shown that stimuli negatively correlated with an outcome are functionally identical to stimuli that signal the nonreinforcement of an explicit excitor. However, it is customary to view the negative correlation procedure as much weaker in its ability to generate inhibition than is the standard Pavlovian conditioned inhibition paradigm. For this reason, the results of Experiment 2 should not be construed as being irreconcilable with a conditioned inhibition account of the results of Experiments 1 and 3.

In contrast to the claim that a Pavlovian association exists between $\mathrm{S}+$ and the outcome, other authors have argued that an $\mathrm{S}+$ functions as a modulator of the outcome representation (Colwill \& Rescorla, 1988). Thus, rather than directly activating the outcome representation in the manner of a Pavlovian stimulus, an $\mathbf{S}+$ is thought to lower the threshold for other associates of the outcome to activate that representation. From this perspective, the effect of treatments found to interfere with S+ control would be attributed to their ability to increase the threshold value for activating the outcome representation. The notion that stimuli may have a modulatory function has proven quite fruitful in understanding how Pavlovian occasion-setters operate (Holland, 1983; Rescorla, 1985). Moreover, it suggests that the pertinent feature of the extinction treatments used here is not the nonreinforcement of an instrumental response per se but that $\mathrm{S}+$ signals a reduction in the strength of the association between some event and the outcome.

The third view regarding the associative structure of discriminative stimuli is that an $\mathrm{S}+$ becomes associated with the $\mathrm{R}-\mathrm{O}$ relation arranged in its presence [i.e., $\mathrm{S}-$ (R-O)]. According to this hierarchical account, the detrimental effects of $S-$ training and signaled extinction on $\mathrm{S}+$ control occur because such training establishes an inhibitory connection between $\mathrm{S}+$ and the $\mathrm{R}-\mathrm{O}$ relation [i.e., $S \dashv(\mathrm{R}-\mathrm{O})]$. To explain the present data, it would have to be argued that this inhibitory learning generalizes more to those $\mathrm{R}-\mathrm{O}$ relations associated with the $\mathrm{S}+$ that involve the same outcome. This argument about differential generalization has already been used to account for the ability of an $\mathrm{S}+$ to transfer to new responses trained with the same outcome (Colwill \& Rescorla, 1990b).

This hierarchical analysis of instrumental learning is attractive for two reasons. Firstly, there is excellent documentation of the presence of excitatory associations between $\mathrm{S}+$ and the $\mathrm{R}-\mathrm{O}$ relation (e.g., Colwill \& Rescorla, 1990b; Rescorla, 1990). Secondly, the hierarchical view would seem to satisfy the constraints that Bonardi (1988) has tried to impose on an analysis of the suppressive effects of an S-. However, there is no evidence to indicate that the kind of inhibitory association suggested by the hierarchical analysis actually occurs during $S$ - training. Instead, the available evidence seems to favor the development of simple binary associations (Bonardi, 1989; Colwill, 1991). Thus, to appreciate fully the merits of the hierarchical account, two issues need to be addressed. First, it is important to determine whether or not $\mathbf{S}$ - training does involve hierarchical associations. Second, it is important to clarify the circumstances under which binary and hierarchical associations may interact.

What is clear about the reduction in S+ control observed in Experiments 1 and 3 is that it should discourage appeal to excitatory $S-R$ associations to explain performance of the original response. Two findings have tended to foster support for the view that $S+s$ are directly associated with their instrumental responses. First, the observation of residual responding following devaluation of the outcome appears to suggest that performance of an instrumental response is not entirely determined by the value of the anticipated consequences. However, Colwill and Rescorla (1990a) have reported complete elimination of an instrumental response in the presence of its $\mathrm{S}+$ following outcome devaluation. In their study, the outcome was delivered directly into the oral cavity throughout initial instrumental training and during the devaluation phase. They offer several suggestions as to why direct administration of the outcome leaves no residual responding following devaluation of that outcome. Each of those suggestions affords an explanation within an $\mathrm{R}-\mathrm{O}$ framework for the residual responding routinely observed in studies that use a distal delivery of the reinforcer. Consequently, the observation of residual responding following outcome devaluation is not sufficient grounds for the identification of S-R learning.

The second argument that has been made in favor of excitatory S-R associations is based on the ability of animals to solve particular kinds of instrumental discriminations. The classic example of such a problem is one in which two responses are available but each is reinforced in a different $\mathrm{S}+$. Because the same outcome is used for both responses it has been assumed that the successful solution of this task may only be accomplished with an excitatory S-R association between S+ and the correct response (Mackintosh, 1983). However, it is equally plausible that the solution to such tasks may involve inhibitory S-R associations between each $\mathrm{S}+$ and the incorrect response. Moreover, recent evidence seems to favor a strong role for such inhibitory S-R associations in negative instrumental discriminations. For instance, Colwill (1991) has reported that an S- will continue to suppress its original response even after training as an $\mathrm{S}+$ with another response. Furthermore, the degree of that suppression was unaffected by the identity of the outcome used to retrain the $S-$ as an $S+$. This finding suggests 
that the associative structure mediating $S$ - control is independent of outcome identity and may best be understood in terms of an inhibitory S-R association.

Finally, it is worth noting that the present results and those of Colwill (1991) suggest some asymmetry in the associative structures mediating performance of the original response in positive and negative instrumental discriminations. Whereas $\mathrm{S}-\mathrm{O}$ associations appear to dominate control of the original response in the presence of its $\mathrm{S}+$, inhibitory $\mathrm{S}-\mathrm{R}$ associations appear to be the major determinant of performance of the original response in the presence of its $\mathbf{S}-$. This asymmetry is intriguing because other studies that model instrumental discriminative stimuli with Pavlovian facilitators and inhibitors have not uncovered such a relation. Using decremental manipulations analogous to those reported here, Rescorla (1986) found that a facilitator that signals a positive stimulus-outcome relation may be extinguished by treatments that establish it as a signal for the nonreinforcement of another stimulus trained with that outcome. Similarly, Rescorla (1987) found that the ability of a Pavlovian inhibitor to suppress responding to an excitor was attenuated by training that stimulus as a facilitator but not by training it as a simple excitor. However, the implications of these results may not be fully appreciated until they have been shown to be dependent upon the identity of the outcome.

In summary, the present study has demonstrated that the outcome information provided by an $\mathrm{S}+$ influences the control exerted by that $S+$ on the response earning that outcome. Although the exact nature of this outcome encoding by the $\mathrm{S}+$ remains controversial, there is no doubt that any account of instrumental performance that views positive discriminative stimuli simply as elicitors of their instrumental responses is not viable.

\section{REFERENCES}

AMSEL, A. (1962). Frustrative nonreward in partial reinforcement and discrimination learning: Some recent history and a theoretical extension. Psychological Review, 69, 306-328.

BoNARDI, C. (1988). Mechanisms of inhibitory discriminative control. Animal Learning \& Behavior, 16, 445-450.

BONARDI, C. (1989). Inhibitory discriminative control is specific to both the response and the reinforcer. Quarterly Journal of Experimental Psychology, 41B, 225-242.

CAPALDI, E. J. (1970). An analysis of the role of reward and reward magnitude in instrumental learning. In J. H. Reynierse (Ed.), Current issues in animal learning (pp. 357-389). Lincoln: University of Nebraska Press.

ColWILl, R. M. (1991). Negative discriminative stimuli provide information about the identity of omitted response-contingent outcomes. Animal Learning \& Behavior, 19, 326-336.

Colwill, R. M., \& Rescorla, R. A. (1988). Associations between the discriminative stimulus and the reinforcer in instrumental learning. Journal of Experimental Psychology: Animal Behavior Processes, 14, 155-164:
Colwill, R. M., \& Rescorla, R. A. (1990a). Effect of reinforcer devaluation on discriminative control of instrumental behavior. Journal of Experimental Psychology: Animal Behavior Processes, 16, 40-47.

Colwill, R. M., \& Rescorla, R. A. (1990b). Evidence for the hierarchical structure of instrumental learning. Animal Learning \& Behavior, 18, 71-82.

Hearst, E., \& Franklin, S. R. (1977). Positive and negative relations between a signal and food: Approach-withdrawal behavior. Journal of Experimental Psychology: Animal Behavior Processes, 3, 37-52.

Holland, P. C. (1983). "Occasion-setting" in Pavlovian feature positive discriminations. In M. L. Commons, R. J. Herrnstein, \& A. R. Wagner (Eds.), Quantitative analyses of behavior: Vol IV. Discrimination processes (pp. 183-206). Cambridge, MA: Ballinger.

Hull, C. L. (1943). Principles of behavior. New York: AppletonCentury-Crofts.

LoLordo, V. M., \& FaIrLess, J. L. (1985). Pavlovian conditioned inhibition: The literature since 1969. In R. R. Miller \& N. E. Spear (Eds.), Information processing in animals: Conditioned inhibition (pp. 1-49). Hillsdale, NJ: Erlbaum.

Lovigond, P. F. (1983). Facilitation of instrumental behavior by a Pavlovian appetitive conditioned stimulus. Journal of Experimental Psychology: Animal Behavior Processes, 9, 225-247.

MACKINTOSH, N. J. (1983). Conditioning and associative learning. Oxford: Oxford University Press.

RESCORLA, R. A. (1969). Conditioned inhibition of fear resulting from negative CS-US contingencies. Journal of Comparative \& Physiological Psychology, 67, 504-509.

Rescorla, R. A. (1979). Conditioned inhibition and extinction. In A. Dickinson \& R. A. Boakes (Eds.), Mechanisms of learning and motivation (pp. 83-110). Hillsdale, NJ: Erlbaum.

Rescorla, R. A. (1985). Inhibition and facilitation. In R. R. Miller \& N. E. Spear (Eds.), Information processing in animals: Conditioned inhibition. Hillsdale, NJ: Erlbaum.

ResCORLA, R. A. (1986). Extinction of facilitation. Journal of Experimental Psychology: Animal Behavior Processes, 12, 16-24.

Rescorla, R. A. (1987). Facilitation and inhibition. Journal of Experimental Psychology: Animal Behavior Processes, 13, 250-259.

Rescorla, R. A. (1990). Evidence for an association between the discriminative stimulus and the response-outcome association in instrumental learning. Journal of Experimental Psychology: Animal Behavior Processes, 16, 326-344.

ResCoRlA, R. A., \& LoLCRDo, V. M. (1965). Inhibition of avoidance behavior. Journal of Comparative \& Physiological Psychology, 59, 406-412.

ResCorla, R. A., \& Solomon, R. L. (1967). Two-process learning theory: Relationships between Pavlovian conditioning and instrumental learning. Psychological Review, 74, 151-182.

RoBbiNs, S. J. (1990). Mechanisms underlying spontaneous recovery in autoshaping. Journal of Experimental Psychology: Animal Behavior Processes, 7, 175-190.

Trapold, M. A., \& Overmier, J. B. (1972). The second learning process in instrumental learning. In A. A. Black \& W. F. Prokasy (Eds.), Classical conditioning: II. Current research and theory (pp. 427-452). New York: Appleton-Century-Crofts.

Weisman, R. G., \& Litner, J. S. (1969). Positive conditioned reinforcement of Sidman avoidance behavior in rats. Joumal of Comparative \& Physiological Psychology, 68, 597-603.

Wielkiewicz, R. M. (1979). Effects of CSs for food and water upon rats barpressing for different magnitudes of food reinforcement. $A n$ imal Learning \& Behavior, 7, 246-250.

(Manuscript received March 12, 1992; revision accepted for publication March 23, 1993.) 